\title{
Front Matter: Volume 8980
}

, "Front Matter: Volume 8980," Proc. SPIE 8980, Physics and Simulation of Optoelectronic Devices XXII, 898001 (28 March 2014); doi:

$10.1117 / 12.2063466$

SPIE. Event: SPIE OPTO, 2014, San Francisco, California, United States 


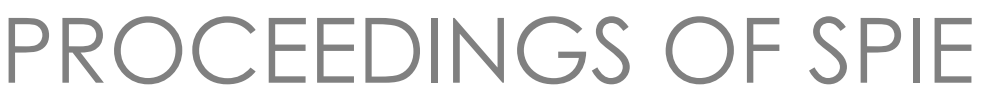

\title{
Physics and Simulation of Optoelectronic Devices XXII
}

\author{
Bernd Witzigmann \\ Marek Osiński \\ Fritz Henneberger \\ Yasuhiko Arakawa \\ Editors
}

3-6 February 2014

San Francisco, California, United States

Sponsored and Published by

SPIE 
The papers included in this volume were part of the technical conference cited on the cover and title page. Papers were selected and subject to review by the editors and conference program committee. Some conference presentations may not be available for publication. The papers published in these proceedings reflect the work and thoughts of the authors and are published herein as submitted. The publisher is not responsible for the validity of the information or for any outcomes resulting from reliance thereon.

Please use the following format to cite material from this book:

Author(s), "Title of Paper," in Physics and Simulation of Optoelectronic Devices XXII, edited by Bernd Witzigmann, Marek Osinski, Fritz Henneberger, Yasuhiko Arakawa, Proceedings of SPIE Vol. 8980 (SPIE, Bellingham, WA, 2014) Article CID Number.

ISSN: 0277-786X

ISBN: 9780819498939

Published by

SPIE

P.O. Box 10, Bellingham, Washington 98227-0010 USA

Telephone +1 3606763290 (Pacific Time) · Fax +1 3606471445

SPIE.org

Copyright (@ 2014, Society of Photo-Optical Instrumentation Engineers.

Copying of material in this book for internal or personal use, or for the internal or personal use of specific clients, beyond the fair use provisions granted by the U.S. Copyright Law is authorized by SPIE subject to payment of copying fees. The Transactional Reporting Service base fee for this volume is $\$ 18.00$ per article (or portion thereof), which should be paid directly to the Copyright Clearance Center (CCC), 222 Rosewood Drive, Danvers, MA 01923. Payment may also be made electronically through CCC Online at copyright.com. Other copying for republication, resale, advertising or promotion, or any form of systematic or multiple reproduction of any material in this book is prohibited except with permission in writing from the publisher. The CCC fee code is 0277-786X/14/\$18.00.

Printed in the United States of America.

Publication of record for individual papers is online in the SPIE Digital Library.

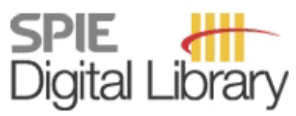

SPIEDigitalLibrary.org

Paper Numbering: Proceedings of SPIE follow an e-First publication model, with papers published first online and then in print and on CD-ROM. Papers are published as they are submitted and meet publication criteria. A unique, consistent, permanent citation identifier (CID) number is assigned to each article at the time of the first publication. Utilization of CIDs allows articles to be fully citable as soon as they are published online, and connects the same identifier to all online, print, and electronic versions of the publication. SPIE uses a six-digit CID article numbering system in which:

- The first four digits correspond to the SPIE volume number.

- The last two digits indicate publication order within the volume using a Base 36 numbering

system employing both numerals and letters. These two-number sets start with 00, 01, 02, 03, 04, $05,06,07,08,09,0 A, 0 B \ldots 0 Z$, followed by 10-1Z, 20-2Z, etc.

The CID Number appears on each page of the manuscript. The complete citation is used on the first page, and an abbreviated version on subsequent pages. Numbers in the index correspond to the last two digits of the six-digit CID Number. 


\section{Contents}

xi Conference Committee

SESSION 1 LIGHT EMITIING DIODES

898003 Monte Carlo-drift-diffusion simulation of electron current transport in III-N LEDs [8980-2]

P. Kivisaari, T. Sadi, J. Oksanen, J. Tulkki, Aalto Univ. (Finland)

\section{SESSION 2 ELECTROMAGNETICS I}

898005 High-bandwidth, low loss suspended silica splitters [8980-5]

S. Soltani, A. M. Armani, The Univ. of Southern California (United States)

898006 Topology-optimized broadband surface relief transmission grating [8980-6] J. Andkjaer, C. P. Ryder, P. C. Nielsen, T. Rasmussen, K. Buchwald, Ibsen Photonics A/S (Denmark); O. Sigmund, Technical Univ. of Denmark (Denmark)

898007 Optical modelling of incoherent substrate light-trapping in silicon thin film multi-junction solar cells with finite elements and domain decomposition [8980-7]

M. Hammerschmidt, Konrad-Zuse-Zentrum für Informationstechnik Berlin (Germany); D. Lockau, Konrad-Zuse-Zentrum für Informationstechnik Berlin (Germany) and HelmholtzZentrum Berlin für Materialien und Energie GmbH (Germany); L. Zschiedrich, F. Schmidt, Konrad-Zuse-Zentrum für Informationstechnik Berlin (Germany) and JCMwave GmbH (Germany)

898008 Use of a hybrid ray-thin film interference model for the optimization of a FTIR FOEWS [8980-8]

J. R. Godin, P. Nieva, Univ. of Waterloo (Canada)

898009 Study of nano-scale ITO top grating of GaN LED [8980-66]

T. Robinson, G. Halpin, California Polytechnic State Univ., San Luis Obispo (United States); $X$. Jin, California Polytechnic State Univ., San Luis Obispo (United States) and Peking Univ. (China); X.-N. Kang, G.-Y. Zhang, Peking Univ. (China)

\section{SESSION 3 LASER DYNAMICS}

8980 OA Relaxation-oscillation-free semiconductor laser with optical feedback (Invited Paper) [8980-9]

D. Lenstra, Technische Univ. Eindhoven (Netherlands)

8980 OB Rate-equation description of multi-mode semiconductor lasers [8980-10] M. Yousefi, Photonic Sensing Solutions (Netherlands); D. Lenstra, Technische Univ. Eindhoven (Netherlands) 
8980 0C Dynamics of a semiconductor laser with frequency shifted feedback [8980-11]

Y. Noblet, J. P. Toomey, D. M. Kane, Macquarie Univ. (Australia)

SESSION 4 METAL NANOCAVITIES

8980 OE Metal-cavity submonolayer quantum-dot surface-emitting microlasers (Invited Paper) [8980-13]

P. Qiao, C.-Y. Lu, Univ. of Illinois at Urbana-Champaign (United States); D. Bimberg, Technische Univ. Berlin (Germany); S. L. Chuang, Univ. of Illinois at Urbana-Champaign (United States)

\section{SESSION $5 \quad$ NANO LASERS}

8980 0J Hybrid metal/semiconductor lasers based on confined Tamm plasmons (Invited Paper) [8980-18]

C. Symonds, G. Lheureux, ILM, CNRS, Univ. Claude Bernard Lyon 1 (France); J. P. Hugonin, J. J. Greffet, Lab. Charles Fabry, Institut d Optique, CNRS, Univ. Paris-Sud (France); S. Azzini, J. Laverdant, ILM, CNRS, Univ. Claude Bernard Lyon 1 (France); A. Lemaître, P. Senellart, Lab. de Photonique et de Nanostructures, CNRS (France); J. Bellessa, ILM, CNRS, Univ. Claude Bernard Lyon 1 (France)

$8980 \mathrm{OL} \quad$ Epsilon-near-zero-slot waveguides and their applications in ultrafast laser beam steering [8980-20]

K. Shi, W. Zhao, Z. Lu, Rochester Institute of Technology (United States)

\section{SESSION $6 \quad$ SEMICONDUCTOR-BASED PLASMONICS}

$8980 \mathrm{ON} \quad$ ZnO as a tunable metal: new surface plasmon polaritons at telecommunication wavelengths (Invited Paper) [8980-22]

S. Kalusniak, S. Sadofev, F. Henneberger, Humboldt-Univ. zu Berlin (Germany)

8980 OP Development of ZnO films for near-IR plasmonics (Invited Paper) [8980-24]

D. C. Look, Wright State Univ. (United States), Wyle (United States), and Air Force Research Lab. (United States); K. D. Leedy, D. L. Agresta, Air Force Research Lab. (United States)

$89800 Q \quad$ Laser processing of conductive oxides for near-IR plasmonics (Invited Paper) [8980-25]

A. Piqué, H. Kim, N. A. Charipar, M. Osofsky, Naval Research Lab. (United States)

\section{SESSION $7 \quad$ III-NITRIDE-BASED OPTOELECTRONICS}

8980 OR Numerical simulation of deep-UV avalanche photodetectors [8980-26]

E. Bellotti, Boston Univ. (United States); F. Bertazzi, Boston Univ. (United States) and IEIIT-CNR, Politecnico di Torino (Italy)

8980 OS Simulation of water photo electrolysis with III-nitride semiconductor nano wires [8980-27] B. Witzigmann, M. Bettenhausen, M. Mewes, H. Fülle, F. Römer, Univ. Kassel (Germany) 
8980 OT Numerical simulation of III-nitride lattice-matched structures for quantum cascade lasers [8980-28]

S. Shishehchi, R. Paiella, E. Bellotti, Boston Univ. (United States)

$8980 \mathrm{OU} \quad$ Numerical modeling of improvement in slope sensitivity of InGaN-based ring laser rotation sensor [8980-29]

H. Kalagara, P. G. Eliseev, The Univ. of New Mexico (United States); M. Osiński, The Univ. of New Mexico (United States)and Univ. Carlos III de Madrid (Spain)

\section{SESSION 8 SEMICONDUCTOR LASERS}

$8980 \mathrm{OV}$ Theoretical and experimental analysis of unidirectionality of asymmetrically coupled semiconductor ring or disk lasers [8980-30]

G. Morthier, P. Mechet, Univ. Gent (Belgium)

8980 OW Simulation of III-V strained quantum well lasers with coupled concentric racetrack resonators [8980-31]

J. Viegas, P. Xing, S. M. Serunjogi, Masdar Institute of Science \& Technology (United Arab Emirates)

8980 0X Temperature dependences of metal-clad subwavelength semiconductor lasers (MCSELs): geometric invariance and the spontaneous emission factor [8980-81]

J. S. T. Smalley, Q. Gu, M. Puckett, Y. Fainman, Univ. of California, San Diego (United States)

8980 OY Modeling of optical gain in GalnNAs quantum wells by using 8-band and 10-band models [8980-33]

M. Gladysiewicz, M. S. Wartak, Wroclaw Univ. of Technology (Poland) and Wilfrid Laurier Univ. (Canada)

\section{SESSION $9 \quad$ PLASMONICS}

$8980 \mathrm{OZ}$ Plasmonic enhancement and losses in light-emitting quantum-well structures incorporating metallic gratings [8980-34]

T. Sadi, J. Oksanen, J. Tulkki, Aalto Univ. (Finland)

898010 Analysis of hybridized surface plasmon resonance sensor with metallic nanoparticles for high sensitivity [8980-35]

B.-H. Lee, G.-Y. Oh, H.-S. Kim, T.-K. Lee, Chung-Ang Univ. (Korea, Republic of); D.-G. Kim, Korea Photonics Technology Institute (Korea, Republic of); Y.-W. Choi, Chung-Ang Univ. (Korea, Republic of)

898011 Enhancement of Goos-Hänchen effect in a prism-waveguide coupling system with magneto-optic material [8980-36]

T. Tang, Univ. of Electronic Science and Technology of China (China) and Chengdu Univ. of Information Technology (China); L. Deng, J. Qin, L. Bi, Univ. of Electronic Science and Technology of China (China)

898012 Tunable plasmonic metamaterial based on transparent conducting oxide [8980-37]

K. Shi, R. R. Haque, W. Zhao, R. Zhao, Z. Lu, Rochester Institute of Technology (United States) 
898013 Optimizing light absorption in a thin-film p-i-n solar cell using a quasi-periodic grating [8980-38]

M. R. M. Atalla, The Pennsylvania State Univ. (United States)

\section{SESSION $11 \quad$ LASER INJECTION LOCKING}

898014 Rate equation analysis of frequency chirp in optically injection-locked quantum cascade lasers [8980-40]

C. Wang, LTCI, CNRS, Telecom Paristech, Ecole Nationale Supérieure des

Télécommunications (France) and CNRS FOTON, INSA, Univ. Européenne de Bretagne (France); F. Grillot, LTCI, CNRS, Telecom Paristech, Ecole Nationale Supérieure des Télécommunications (France); V. I. Kovanis, The Ohio State Univ. (United States) and Air Force Research Lab. (United States); J. D. Bodyfelt, Massey Univ. Albany (New Zealand); J. Even, INSA, CNRS FOTON, Univ. Européenne de Bretagne (France)

898015 Effect of light backscattering on high-speed modulation performance in strongly injectionlocked unidirectional semiconductor ring lasers [8980-41]

G. A. Smolyakov, The Univ. of New Mexico (United States); M. Osiński, The Univ. of New Mexico (United States)and Univ. Carlos III de Madrid (Spain)

898016 Experimental and simulation analysis of stimulated Brillouin scattering in continuous wave regime [8980-42]

R. Sanchez-Lara, J. A. Alvarez-Chávez, Ctr. de Investigación e Innovación Tecnológica (Mexico); L. de la Cruz-May, F. Méndez Martinez, Univ. Autónoma del Carmen (Mexico)

898017 Reflective semiconductor optical amplifier submitted to strong optical feedback and coupled to long external cavity [8980-43]

M. Ruiz, P. Besnard, T. Chartier, CNRS FOTON (France) and Univ. Européenne de Bretagne, Univ. de Rennes I (France); F. Saliou, S. D. Le, Q. Deniel, P. Chanclou, Orange Labs., France Telecom (France)

898018 Numerical analysis of frequency chirp in strongly injection-locked semiconductor ring lasers [8980-44]

F.-H Chu, G. A. Smolyakov, The Univ. of New Mexico (United States); M. Osiński, The Univ. of New Mexico (United States) and Univ. Carlos III de Madrid (Spain)

\section{SESSION 12 GRAPHENE OPTOELECTRONICS}

$89801 \mathrm{~A}$ Difference frequency generation of terahertz surface plasmons in graphene and topological insulators [8980-46]

X. Yao, Texas A\&M Univ. (United States); M. Tokman, Institute of Applied Physics (Russian Federation); A. Belyanin, Texas A\&M Univ. (United States) 
8980 1B Electronic band structure and photoemission spectra of graphene on silicon substrate [8980-47]

B. Javvaji, Indian Institute of Science (India); A. Ravikumar, Indian Institute of Science (India) and National Institute of Technology, Surathkal (India); B. M. Shenoy,

D. Roy Mahapatra, Indian Institute of Science (India); M. R. Rahman, National Institute of Technology, Surathkal (India); G. M. Hegde, Indian Institute of Science (India)

8980 1C Novel approaches to enhance graphene absorption and electro-optic property [8980-48] K. Shi, W. Zhao, B. Zhao, Z. Lu, Rochester Institute of Technology (United States)

\section{SESSION 13 ELECTROMAGNETICS II}

8980 1E Non-reciprocal optical devices based on linear silicon photonic crystals [8980-50]

D. Franco Rêgo, V. F. Rodriguez-Esquerre, Univ. Federal da Bahia (Brazil)

$8980 \mathrm{IF} \quad$ Supersymmetric optical waveguides [8980-51]

M.-A. Miri, M. Heinrich, D. N. Christodoulides, CREOL, The College of Optics and Photonics, Univ. of Central Florida (United States)

8980 IG Multimode interferometers based on non-conventional waveguides [8980-52]

A. J. R. F. de Oliveira, Univ. Federal da Bahia (Brazil) and Univ. Federal do Vale do São Francisco (Brazil); V. F. Rodriguez-Esquerre, Univ. Federal da Bahia (Brazil)

$8980 \mathrm{1H} \quad$ Ultra-thin low loss Si3N4 optical waveguides at $1310 \mathrm{~nm}$ [8980-53]

S. T. Lim, A. Gandhi, C. E. Png, Institute of High Performance Computing (Singapore); D. Lu, N.S. S. Ang, E. J. Teo, J. Teng, Institute of Materials Research and Engineering (Singapore)

\section{SESSION 14 QUANTUM DOT LASERS}

898011 Group-velocity slowdown in quantum-dots and quantum-dot molecules (Invited Paper) [8980-54]

S. Michael, Technishe Univ. Kaiserslautern (Germany); W. W. Chow, Sandia National Labs. (United States); H. C. Schneider, Technishe Univ. Kaiserslautern (Germany)

8980 i J Monte Carlo markovian modeling of modal competition in dual-wavelength semiconductor lasers [8980-55]

L. Chusseau, IES, CNRS, Univ. Montpellier 2 (France); F. Philippe, A. Jean-Marie, LIRMM, CNRS (France) 
$89801 \mathrm{~K}$ Nondegenerate four-wave mixing in a dual mode injection locked quantum dot laser [8980-56]

F. Grillot, LTCl, CNRS, Telecom Paristech, Ecole Nationale Supérieure des

Télécommunications (France); C. Wang, LTCl, CNRS, Telecom Paristech, Ecole Nationale Supérieure des Télécommunications (France) and INSA, CNRS FOTON, Univ. Européenne de Bretagne (France); I. A. Aldaya-Garde, LTCl, CRNS, Telecom Paristech, Ecole Nationale Supérieure des Télécommunications (France) and Instituto Tecnológico y de Estudios Speriores de Monterrey (Mexico); C. Gosset, LTCl, CRNS, Telecom Paristech, Ecole Nationale Supérieure des Télécommunications (France); T. Batte, INSA, CNRS FOTON, Univ. Européenne de Bretagne (France); E. Decerle, Yenista Optics (France); J. Even, INSA, CNRS FOTON, Univ. Européenne de Bretagne (France)

$8980 \mathrm{lL} \quad$ Ultra-strongly sub-Poissonian light generation in a quantum dot-bimodal cavity system [8980-57]

W. Zhang, Z. YU, Y. Liu, Y. Peng, Beijing Univ. of Posts and Telecommunications (China)

\section{POSTER SESSION}

$89801 \mathrm{M}$ Carrier transport in dichromatic color-coded semipolar (2021) and (2021) III-N LEDs [8980-3]

M. V. Kisin, C. L. Huang, H. S. El-Ghoroury, Ostendo Technologies, Inc. (United States)

8980 iN Generation of pulse trains with high-repetition-rate in anomalous dispersion decreasing fibers [8980-59]

D. A. Korobko, Ulyanovsk State Univ. (Russian Federation); O. G. Okhotnikov, Tampere Univ. of Technology (Finland); A. A. Sysoliatin, A. M. Prokhorov General Physics Institute (Russian Federation); I. O. Zolotovskii, Ulyanovsk State Univ. (Russian Federation)

898010 Adaptive sampling strategies for efficient parameter scans in nano-photonic device simulations [8980-60]

M. Hammerschmidt, Konrad-Zuse-Zentrum für Informationstechnik Berlin (Germany); J. Pomplun, JCMwave GmbH (Germany); S. Burger, F. Schmidt, Konrad-Zuse-Zentrum für Informationstechnik Berlin (Germany) and JCMwave GmbH (Germany)

8980 IP Modeling of opto-electronics in complex photonic integrated circuits [8980-61]

C. Arellano, VPIphotonics GmbH (Germany); S. Mingaleev, VPI Development Ctr. (Belarus); I. Koltchanov, A. Richter, VPIphotonics GmbH (Germany)

$89801 Q \quad$ Toward bound-to-continuum photon absorption with quantum tunneling in type-II nanostructures: a source-radiation scheme using perfectly-matched layers [8980-62] C.-T. Hsieh, Academia Sinica (Taiwan); S.-W. Chang, Academia Sinica (Taiwan) and National Chiao Tung Univ. (Taiwan)

8980 1T Design and simulation of an optical waveguide for its integration with a light source based on SRO [8980-65]

J. Alarcón-Salazar I. E. Zaldívar-Huerta, M. Aceves-Mijares, Instituto Nacional de Astrofísica, Óptica y Electrónica (Mexico) 
$89801 \mathrm{~V}$ GPGPU-based parallel computing of PIC-FDTD simulation for the development of novel terahertz radiation devices [8980-68]

T. Iwata, A. Okajima, T. Matsui, Mie Univ. (Japan)

$89801 \mathrm{~W}$ Graphical computational method for active materials in simulation of optical electromagnetics [8980-70]

M. Potasek, E. Parilov, K. Beeson, Simphotek Inc. (United States)

$89801 \mathrm{X}$ Impact of the gain model on the stability assessment in semiconductor DFB lasers [8980-71] I. Aldaya, G. Campuzano, Tecnológico de Monterrey (Mexico); C. Gosset, C. Wang, F. Grillot, LTCl, CNRS, Télécom ParisTech (France); G. Castañón, Tecnológico de Monterrey (Mexico)

8980 1Y Plasmonic gap mode nanocavities at telecommunication wavelengths [8980-72]

P.-J. Cheng, National Chiao Tung Univ. (Taiwan) and Academia Sinica (Taiwan);

C.-Y. Weng, Academia Sinica (Taiwan) and National Taiwan Ocean Univ. (Taiwan);

S.-W. Chang, National Chiao Tung Univ. (Taiwan) and Academia Sinica (Taiwan); T.-R. Lin, National Taiwan Ocean Univ. (Taiwan); C.-H. Tien, National Chiao Tung Univ. (Taiwan)

$898021 \quad$ Characteristics of microwave frequency combs generated by a semiconductor laser under dynamical dual-beam injection [8980-75]

C.-T. Lin, Y.-S. Juan, Yuan Ze Univ. (Taiwan)

898022 Physical modeling of RF source generation based on electro-optic modulation and laser injection locking [8980-76]

S. Shi, G. Schneider, D. W. Prather, Univ. of Delaware (United States)

898023 Analysis of evanescent fiber optic sensors using Meep as a simulation tool [8980-77]

L. Zdravkova, P. Nieva, Univ. of Waterloo (Canada)

$898024 \quad$ Highly strained $\ln _{\mathbf{x}} \mathbf{G a}_{1-\mathrm{x}} \mathbf{A} \mathbf{s}_{1-y} \mathrm{Sb}_{\mathrm{y}} / \mathrm{GaSb}$ for mid-infrared devices [8980-78]

C. Meyer, J. Grayer, D. Paterson, E. Cheng, G. Triplett, Univ. of Missouri-Columbia (United States)

898027 Thermal considerations in electrically pumped metallo-dielectric nanolasers [8980-82] J. Shane, Q. Gu, Univ. of California, San Diego (United States); F. Vallini, Univ. Estadual de Campinas (Brazil); B. Wingad, J. S. T. Smalley, Univ. of California, San Diego (United States);

N. C. Frateschi, Univ. Estadual de Campinas (Brazil); Y. Fainman, Univ. of California, San Diego (United States)

Author Index 


\section{Conference Committee}

Symposium Chairs

David L. Andrews, University of East Anglia Norwich (United Kingdom)

Alexei L. Glebov, OptiGrate Corporation (United States)

Symposium Cochairs

Jean Emmanuel Broquin, IMEP-LAHC (France)

Shibin Jiang, AdValue Photonics, Inc. (United States)

Program Track Chair

James G. Grote, Air Force Research Laboratory (United States)

Conference Chairs

Bernd Witzigmann, Universität Kassel (Germany)

Marek Osiński, The University of New Mexico (United States)

Fritz Henneberger, Humboldt- Universität zu Berlin (Germany)

Yasuhiko Arakawa, The University of Tokyo (Japan)

Conference Program Committee

Hiroshi Amano, Nagoya University (Japan)

Toshihiko Baba, Yokohama National University (Japan)

Weng W. Chow, Sandia National Laboratories (United States)

Shun Lien Chuang, University of Illinois at Urbana-Champaign (United States)

Aldo Di Carlo, Università degli Studi di Roma Tor Vergata (Italy)

Keiichi Edamatsu, Tohoku University (Japan)

Nicholas J. Ekins-Daukes, Imperial College London (United Kingdom)

Alexandre Freundlich, University of Houston (United States)

Ortwin Hess, Imperial College London (United Kingdom)

Stephan W. Koch, Philipps- Universität Marburg (Germany)

Vassillios I. Kovanis, Air Force Research Laboratory (United States)

Nikolay N. Ledentsov, VI Systems GmbH (Germany)

Cun-Zheng Ning, Arizona State University (United States)

Joachim Piprek, NUSOD Institute LLC (United States)

Ikuo Suemune, Hokkaido University (Japan) 


\section{Session Chairs}

$1 \quad$ Light Emitting Diodes

Bernd Witzigmann, Universität Kassel (Germany)

2 Electromagnetics I

Thomas A. Klar, Johannes Kepler Universität Linz (Austria)

3 Laser Dynamics

Ikuo Suemune, Hokkaido University (Japan)

4 Metal Nanocavities

Geert Morthier, Universiteit Gent (Belgium)

5 Nano Lasers

Cun-Zheng Ning, Arizona State University (United States)

6 Semiconductor-based Plasmonics

Yasuhiko Arakawa, The University of Tokyo (Japan)

$7 \quad$ III-Nitride-based Optoelectronics

Fritz Henneberger, Humboldt- Universität zu Berlin (Germany)

8 Semiconductor Lasers

Nikolay N. Ledentsov, VI Systems GmbH (Germany)

9 Plasmonics

Friedhard Römer, Universität Kassel (Germany)

10 Photovoltaics Modeling: Joint Session with Conferences 8980 and 8981

Alexandre Freundlich, University of Houston (United States)

Bernd Witzigmann, Universität Kassel (Germany)

11 Laser Injection Locking

Enrico Bellotti, Boston University (United States)

12 Graphene Optoelectronics

Hans Christian Schneider, Technische Universität Kaiserslautern (Germany)

13 Electromagnetics II

Laurent Chusseau, Université Montpellier 2 (France)

14 Quantum Dot Lasers

Daan Lenstra, Technische Universiteit Eindhoven (Netherlands) 